\title{
La publicación de casos clínicos desde la perspectiva del revisor. Una experiencia necesaria, formativa y gratificante
}

Los casos clínicos se consideran como el primer escalón en la comunicación científica en Medicina al tratarse de un tipo de evidencia anecdótica'. Son numerosas las revistas y los congresos que no admiten este tipo de trabajo para su difusión. Ello no significa, ni mucho menos, que carezcan de valor. Ya Sir William Osler, padre de la Medicina Interna, consideraba un deber del clínico el registrar y comunicar de forma clara aquellos hallazgos inusuales con los que se encontrase en su trabajo asistencial².

Los casos clínicos constituyen una herramienta formativa útili,4 tanto para el que los lee, como para el que los escribe y, por supuesto, para el que tiene que revisarlos previamente para su publicación. A esta última tarea hemos dedicado nuestros esfuerzos desde hace muchos años en diferentes revistas tanto en castellano como en inglés y desde aquí agradezco a sus editores la confianza depositada.

Podría pensarse que los casos clínicos deben estar dedicados únicamente a comunicar el hallazgo de una enfermedad extraordinariamente rara o poco divulgada. Siendo este uno de los posibles objetivos de estos artículos, no es el único y ni siquiera nos parece el más importante para la formación de los médicos al ser altamente improbable que se tengan que enfrentar con esa enfermedad a lo largo de su ejercicio profesional.

A nuestro juicio, será de mayor utilidad comunicar síntomas o signos inusuales de una enfermedad común o efectos adversos no descritos de un medicamento de amplio uso. También pueden ser objeto de los casos clínicos tratamientos fuera de las indicaciones autorizadas 5 . Por otro lado, el caso clínico no se circunscribe al campo de la Patología pudiendo dar cuenta de una variación posicional o cuantitativa de las estructuras anatómicas.

Como apuntábamos al principio de estas líneas, varias revistas han proscrito de sus páginas los casos clínicos. Frente a ello no son pocas las publicaciones dedicadas en exclusiva a este tipo de trabajos científicos o revistas de alto impacto como New England Journal of Medicine o The Lancet que los mantienen como uno de sus apartados más señeros.

Basándose en la existencia de recomendaciones para la elaboración de ensayos clínicos randomizados (Consolidated Standards of Reporting Trials, CONSORT), estudios observacionales (Strengthening the Reporting of Observational studies in Epide- miology, STROBE) y revisiones sistemáticas (Preferred Reporting Items for Systematic Reviews and Meta-Analyses, PRISMA), un grupo de clínicos, investigadores y editores de revistas científicas se reunieron en octubre de 2012 para consensuar unos requisitos para comunicar apropiadamente casos clínicos ${ }^{6}$. Inicialmente valoraban trece aspectos, desde el título al consentimiento informado, a los que se añadió posteriormente uno más ${ }^{7}$ analizando un total de treinta y un ítems.

Estas guías, a modo de checklist conocidas como criterios CARE (CAse REport) son de gran utilidad tanto para el autor como para el revisor por pares, siendo recomendadas explícitamente por algunas revistas como nuestra RECCMI, BM Case Report o JAMA

A la hora de enfrentarse a un caso clínico enviado para su publicación en una revista científica se debe ser riguroso al enjuiciar tanto su contenido como su presentación. Pero no debemos perder de vista que habitualmente estamos ante las primeras producciones científicas de sus autores. Por ello nuestras críticas deben ser siempre constructivas, sabedores de que estos autores noveles pueden tener por delante una larga carrera científica y que nuestros consejos van a encontrar una mente abierta al aprendizaje, pero que pueden no contar todavía con el suficiente bagaje vital y profesional para encajar una crítica severa.

El trabajo de revisor por pares de casos clínicos tiene algunas peculiaridades con respecto a la revisión de otro tipo de artículos científicos. Así, no será necesario prestar atención a los habituales puntos críticos de los ensayos clínicos que se concentran en el apartado de material y métodos como el diseño del estudio, los criterios de inclusión, el tamaño muestral, o las herramientas estadísticas empleadas. Por el contrario, la revisión de la literatura debe ser minuciosa para comprobar que efectivamente nos encontramos ante un hallazgo realmente inusual. Esta tarea que requiere un tiempo del que no siempre disponemos nos ayuda a refrescar nuestros conocimientos y a ponernos al día de las más variadas afecciones, métodos diagnósticos y tratamientos.

Solo me resta animar a los jóvenes lectores de esta revista a iniciarse en el estimulante mundo de la producción científica, adaptando en lo posible sus trabajos a las recomendaciones del CARE, y a los no tan jóvenes a colaborar como peer review. Comprobarán tanto unos como otros que los frutos merecen el esfuerzo empleado.

Víctor José González-Ramallo Unidad de Hospitalización a Domicilio, Servicio de Medicina Interna, Hospital General Universitario Gregorio Marañón. Universidad Complutense de Madrid, Madrid, España

vjgonzal@ucm.es

Citar como: González-Ramallo VJ. La publicación de casos clínicos desde la perspectiva del revisor. Una experiencia necesaria, formativa y gratificante. Rev Esp Casos Clin Med Intern (RECCMI). 2021 (dic); 6(3): 1-2. doi: 10.32818/reccmi.a6n3a1.

Cite this as: González-Ramallo VJ. The publication of clinical cases from the reviewer's perspective. A necessary, formative and rewarding experience. Rev Esp Casos Clin Med Intern (RECCMI). 2021 (Dec); 6(3): 1-2. doi: 10.32818/reccmi.a6n3a1. 


\section{Bibliografía}

1. Riley D. Case reports in the era of clinical trials. Glob Adv Health Med. 2013 2(2): 10-11. doi: 10.7453/gahmj.2013.012.

2. Cabán-Martínez AJ, García-Beltrán WF. Advancing medicine one research note at a time: the educational value in clinical case reports. BMC Res Notes. 2012; 5: 293. doi: 10.1186/1756-0500-5-293.

3. Mellado JM, Packer CD. The clinical case: Validity, values and strategies to approach its writing. Radiología. 2017; 59: 496-503. doi: 10.1016/j. rx.2017.07.002

4. Vandenbroucke J. In defense of case reports and case series. Ann Intern Med. 2001; 134(4): 330-334. doi: 10.7326/0003-4819-134-4-200102200-00017.
5. Sanjurjo-Sáez M, Lorenzo-Pinto A. La importancia de los casos clínicos para la Farmacia Hospitalaria. Rev Esp Casos Clin Med Intern (RECCMI). 2021; 6(2): 1. doi: 10.32818/reccmi.a6n2a1.

6. Gagnier JJ, Kienle G, Altman DG, Moher D, Sox H, Riley D; CARE Group. The CARE guidelines: consensus-based clinical case reporting guideline development. J Med Case Rep. 2013; 7: 223. doi: 10.1186/1752-1947-7-223.

7. Riley DS, Barber MS, Kienle GS, Aronson JK, von Schoen-Angerer T, Tugwell $P$, et al. CARE guidelines for case reports: explanation and elaboration document. J Clin Epidemiol. 2017; 89: 218-235. doi: 10.1016/j.jclinepi.2017.04.026.

8. Calvache JA, Vera-Montoya M, Ordoñez D, Hernandez AV, Altman D, Moher D. Completeness of reporting of case reports in high-impact medical journals. Eur J Clin Invest. 2020; 50(4): e13215. doi: 10.1111/eci.13215. 